EPJ Web of Conferences 110,01027 (2016)

DOI: $10.1051 /$ epjconf/201611001027

(C) Owned by the authors, published by EDP Sciences, 2016

\title{
INFLUENCE PULSE DURATION METHODICAL ERROR OF DETERMINATION OF THERMAL TRANSLUCENT MATERIALS LASER FLASH METHOD
}

\author{
Mark M. Katz ${ }^{1, \text { a }}$, Ilija M.Katz ${ }^{1}$ \\ ${ }^{1}$ National Research Tomsk Polytechnic University, 634050 Tomsk, Russia
}

\begin{abstract}
The analysis of errors in the determination of thermal diffusivity of a typical semiconductor material Germany, due to radiative energy transfer in the heated layer of material, under conditions consistent with the implementation of the method under the influence of the laser pulse on the surface of the collimated laser pulse of finite duration.
\end{abstract}

\section{Introduction}

In recent decades, increased attention is drawn to methods for determining the thermal pulse, the thermal conductivity and heat capacity of the materials at moderate temperatures. The greatest interest in solving these problems is the laser flash method, which is based on the simple implementation of the experimental method and the approximate solution of one-dimensional heat conduction problems [1-2]. Set group of factors [3-7], affecting the value of methodological errors in the determination of thermal characteristics (TPC) materials by laser flash [1].

These factors include the thickness of the samples, the shape and size of the laser heating, heat transfer from the surfaces of the specimen, the spatial nature of heat transfer, thermal conductivity of the material, its porosity and translucency [3-7]. It was also found that the value of the error in determining whether TPC metals depend on the duration of the impulse [3].

However, the influence of the last factor on the accuracy of the determination of the thermal diffusivity of translucent materials has not been investigated. At present, such materials (including semiconductors) are increasingly used in the manufacture of various elements of control systems and space vehicles [8], the reliability of which depends largely on the thermal conditions of their work. The heating process is accompanied by the accumulation of materials such heat supplied not only by conduction, but also the bulk absorption in certain spectral regions, depending on the wavelength of the thermal radiation. It is of interest to analyze the extent of the influence of the pulse duration and translucency material error in the determination of thermal diffusivity by laser flash.

This paper considers the impact of the duration of the laser pulse on the error in the determination of thermal mo-limiting translucent semiconductor material - germanium in the model conductive-radiative heat transfer in the transverse dimensions of the heated surface areas smaller than the transverse dimensions of the sample, in a possible range of real time.

The error was established by comparing the reference values of TPC to certain material as described in [3-7] in numerical modeling of heat transfer in the sample under conditions corresponding to the experiment.

\section{Statement of the problem}

In case of a problem definition it was supposed that material is gray, and own radiation isn't enough in comparison with an external radiation flow. Such assumption doesn't superimpose essential restrictions on a community of setting of the task and real conditions of a heat transfer through translucent material. As a rule, for carrying out experiments by a technique [1] samples are made in the form of disks. The transverse section of a laser beam represents a circle.

For an assessment of scales of influence of processes of radiation heat transfer in a heated-up layer of material the twodimensional task of heat conduction for a disk of the limited sizes in case of impact on a section of its surface of the collimated laser radiation of temporal duration and absence of heat exchange on a section is solved "hot" and all "cold" boundaries (Fig. 1) which aren't heated by laser pulse.

\footnotetext{
${ }^{\mathrm{a}}$ Corresponding author: riki@tpu.ru
} 


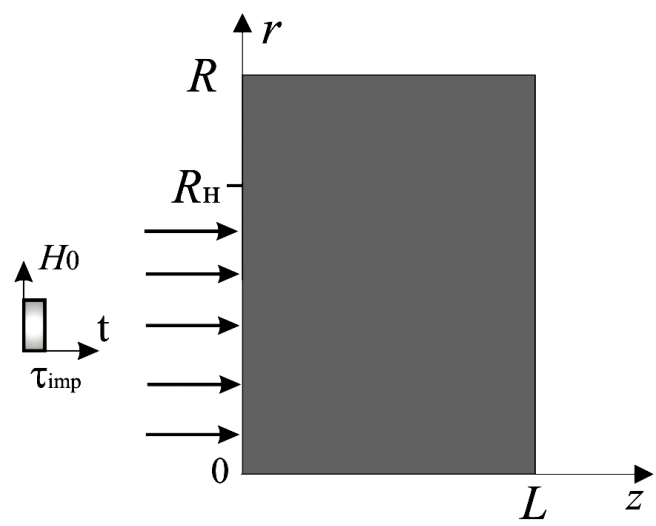

Figure 1. Scheme of solutions domain.

A mathematical model of the process in this case is the energy equation (1) with boundary conditions (2-8):

$$
\begin{gathered}
c \rho \frac{\partial T}{\partial t}=\frac{\lambda}{r} \cdot \frac{\partial}{\partial r}\left(r \frac{\partial T}{\partial r}\right)+\lambda \frac{\partial^{2} T}{\partial z^{2}}-\frac{d H(z)}{d z}-\frac{d H(r)}{d r}, \quad 0<z<L ; 0<r<R \\
z=0: \quad-\lambda\left(\frac{\partial T}{\partial z}\right)=H_{0}, r \leq R_{\mathrm{i}}, 0<t \leq \tau_{\mathrm{imp}} ; \\
z=0: \quad-\lambda\left(\frac{\partial T}{\partial z}\right)=0, R>r>R_{\mathrm{i}}, t>0 ; \\
z=0:-\lambda\left(\frac{\partial T}{\partial z}\right)=0, \quad t>\tau_{\mathrm{imp}} ; \\
z=L:-\lambda\left(\frac{\partial T}{\partial z}\right)=0, \quad 0<r<R, \quad t>0 \\
r=0: \quad-\lambda\left(\frac{\partial T}{\partial r}\right)=0,0<z<L, \quad t>0 ; \\
r=R: \quad-\lambda\left(\frac{\partial T}{\partial r}\right)=0,0<z<L, \quad t>0 ; \\
t=0 ; \quad T=T_{0} .
\end{gathered}
$$

where $c$ - heat capacity; $\rho$ - the density; $\lambda$ - thermal conductivity; $T, T_{0}$ - initial value of the current and temperature; $\mathrm{t}$ - time; - the duration of the laser pulse; $L$ - the thickness and the radius $R$ of the sample; $R_{\mathrm{H}}$ - radius of the cross section of a laser beam.

Changing the energy density of radiationtion of the material was determined by the Bouguer-Lambert-Beer [9]:

$$
\begin{aligned}
& H(z)=H_{0} \cdot \exp \left(-k_{\lambda} \cdot z\right), \\
& H(r)=H(z) \cdot \exp \left(-k_{\lambda} \cdot r\right),
\end{aligned}
$$

where $H(\mathrm{z}), H(\mathrm{r})$ - of the radiation flux density in the material along the coordinate $r$ and $z$ directions; $k_{\lambda}$ - absorption coefficient of the collimated laser radiation flux.

\section{Solution method}

The problem (1) - (9) is solved by finite difference method [10], using the developed solutions for singularly perturbed boundary value problems of heat transfer [11] iterative algorithm $[12,13]$. The validity of the theoretical consequences of controlled test conservativeness difference scheme used by the method of $[14,15]$, designed to support the adequacy of the results of numerical studies of nonlinear processes of heat and mass transfer under intensive physical and chemical transformations in the areas of small size with the release (or absorption) of energy. Steps difference grid by the time chosen as in [16, 17], and the range from $10^{-4}$ to $10^{-7} \mathrm{~s}$ with depending on the heat. 


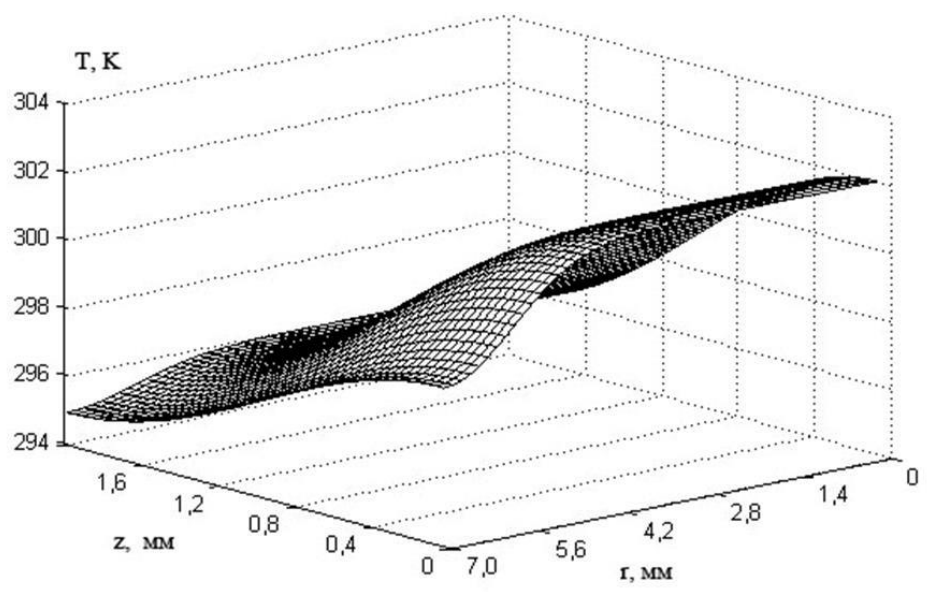

Figure 2. Distribution of $T(z, r)$ in a sample $\left(R=7 \mathrm{~mm}, \tau_{\text {imp }}=1,0 \mathrm{~ms}, \mathrm{k}_{\lambda}=20 \mathrm{~m}^{-1}\right)$ at time $\mathrm{t}=1,5 \mathrm{~ms}$.

\section{Results and discussion}

Numerical modeling of heat transfer process in a transparent semiconductor material carried out by the example of model material-germanium (thermal characteristics: $\left.\lambda=64 \mathrm{~W} /(\mathrm{m} \cdot \mathrm{K}), c=316.2 \mathrm{~J} /(\mathrm{kg} \cdot \mathrm{K}), \rho=5460 \mathrm{~kg} / \mathrm{m}^{3}\right)$ [18]. Numerical analysis is carried out under the following initial data: the value of the initial temperature $\mathrm{T}_{0}=293 \mathrm{~K}$, the sample thickness of $3 \mathrm{~mm}$ (range varies from 5 to $15 \mathrm{~mm}$ ) wavelength range of the region of transparency of germanium is $1800 \ldots 15000 \mathrm{~nm}$ (corresponding to a change in the absorption coefficient $k_{\lambda} 0,5 \ldots 50 \mathrm{~m}-1$ [18]). Terms of heat exposure to the material: the intensity of the heat flow , pulse duration $0.5 \ldots 5 \mathrm{~ms}$. The calculations were performed with moves in space to 3 microns.

Is established (Fig. 2) that the local uniform heat flux in the heating zone temperature changes not only by the thickness of the disc, but also the radial coordinate, which leads to a change in the maximum temperature on the "cold" surfaces in comparison with a one-dimensional problem statement [1 ].

The results lead to the conclusion not substantially one-dimensional distribution of thermal processes in a transparent material, and the impact of the last factor on the error in the determination of thermal diffusivity of the material.

Fig. $3(\mathrm{a}, \mathrm{b})$ shows the calculated after solving the problem (1-9) depending on the thermal error $\left(\delta_{a}\right)$ Germany on the pulse duration when the absorption coefficient 10 and $\mathrm{k}_{\lambda} 20 \mathrm{~m}-1$ and the radius of the heated portion of the sample surface $5,7,15 \mathrm{~mm}$.

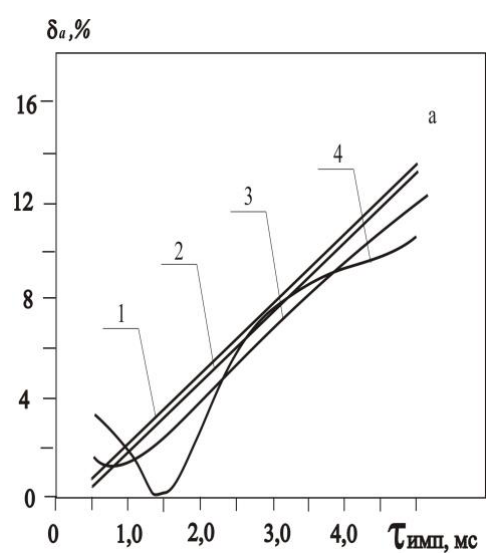

a)

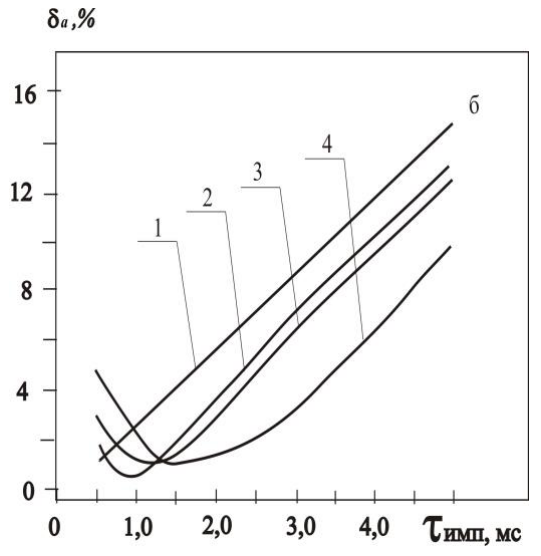

b)

Figure 3. Depending error diffusivity of germanium on the pulse duration of laser heating of the absorption coefficient at $\mathrm{k}_{\lambda}=20$ (a) and 20 (b) $\mathrm{m}^{-1}: 1$ - dimensional problem; $2-R=15 \mathrm{~mm}\left(R_{\mathrm{H}}=0,8 R\right) ; 3-7 ; 4-5$. 


\section{EPJ Web of Conferences}

According to the results of numerical simulation is set (Fig. 3) that the values of the error in determining the thermal diffusivity of germanium depend on the absorption coefficient of the material and the pulse duration of the laser heating. With increasing duration of the laser pulse values monotonically increasing. When $\mathrm{k} \lambda=10 \mathrm{~m}^{-1}$ (Fig. $3 \mathrm{a}$ ), and $\mathrm{R}=7 \mathrm{~mm}$ error amounted to about $2 \%$. When the value of $\mathrm{k} \lambda=20 \mathrm{~m}^{-1}$ (Fig. $3 \mathrm{~b}$ ) and similar amounts of sample error does not exceed $1 \%$.

Suffice significant (Fig. 3) the change in the heat transfer due to the growth of the radial coordinate $r$ both during laser exposure, and after its termination. Accumulated during the pulse in a thin heated to a high temperature sample layer energy during this period is redistributed by conduction and radiation not only coordinate direction $\mathrm{z}$ (heat transfer on which is then used to calculate the thermal diffusivity according to [1]), but also the direction r. Accordingly, the temperature in the "cold" the border would be enough to differ materially from its counterpart in the case of one-dimensional thermal conductivity at the same size "laser spot" and the radius of the sample material.

One major reason is the mismatch error in the determination of the transverse dimensions. The greater the difference between them will be, the more (Fig. 3 a, b).

When identical values of error in the determination of thermal diffusivity is smaller, the larger the radius of the heated portion of the surface of the sample. With increasing RH (under otherwise unchanged conditions) dimensional nature of heat transfer will occur at the point of measurement (at the symmetry axis of the sample) to a lesser extent.

It is known [5] that the inclusion of the possibility of spatial nature of the process of thermal conductivity leads only to increase methodological errors as compared to the one-dimensional heat transfer model. Accordingly, in the present work values are likely to lower (minimum) limit of determination errors.

Analysis of the results (Figure 3) performed theoretical research suggests the possibility of reducing to a minimum of methodological errors in determining TPC translucent materials through Select pulse laser heating at other conditions of adequate thermal experiments.

\section{References}

1. W. J. Parker, R.J. Jenkins , C.P. Butler, J. Appl. Phys., 32 (9), 1675 (1961)

2. O. Altun, B. Erhan, A. Kalemtas, J. Achievements in Materials and Manufacturing Engineering, 30 (2), 115 (2008)

3. G.V. Kuznetsov, M. D. Katz, Meas. Tech., 55 (4), 454 (2012)

4. G.V. Kuznetsov, M. D. Katz, Meas. Tech., 53 (6), 674 (2010)

5. G.V. Kuznetsov, M. D. Katz. Meas. Tech., 52 (4), 674 (2009)

6. M. D. Kats, I. M. Kats, Meas. Tech., 58 (2), 184 (2015)

7. G.V. Kuznetsov, M. D. Katz, Meas. Tech., 55 (9), 1057 (2012)

8. V.S. Kamalov, Manufacturing spacecraft (mechanical engineering, Moscow: Mashinostroenie, 1982)

9. B.V. Yavorsky, F.F. Detlaf, The reverence manual on physics, (Moscow: Nauka, 1979)

10. A.A Samarskiy, Theory the difference schemes (Moscow: Nauka, 1979)

11. A. Chang, F. Hauers, Nonlinear singularly perturbed boundary value problems (Theory and Applications, Moscow: Mir 1988)

12. G.V. Kuznetsov, G.Ya. Mamontov, G. V. Taratushkina, J. Combustion Explosion and shock Waves, 401 (2004)

13. G. V. Kuznetsov, G.Ya. Mamontov G. V. Taratushkina, Khimicheskaya Fizika, 23 (3), 67 (2004)

14. G. V. Kuznetsov, P.A. Strizhak, J. of Engineering Thermophysics, 18 (1), 72 (2008) 